\title{
Evolutionarily Conserved Long
} Non-coding RNA Regulates Gene Expression in Cytokine Storm During COVID-19

\section{OPEN ACCESS}

Edited by:

Mulin Jun Li,

Tianjin Medical University, China

Reviewed by:

Thomas Dandekar,

Julius Maximilian University of

Würzburg, Germany

Angel León-Buitimea,

Autonomous University of Nuevo

León, Mexico

*Correspondence:

Olanrewaju B. Morenikeji

omorenik@hamilton.edu

Bolaji N. Thomas

bntsbi@rit.edu

Specialty section:

This article was submitted to

Synthetic Biology,

a section of the journal

Frontiers in Bioengineering and

Biotechnology

Received: 20 July 2020 Accepted: 27 October 2020 Published: 15 January 2021

Citation:

Morenikeji OB, Bernard K, Strutton E, Wallace $M$ and Thomas BN (2021)

Evolutionarily Conserved Long

Non-coding RNA Regulates Gene

Expression in Cytokine Storm During

COVID-19.

Front. Bioeng. Biotechnol. 8:582953.

doi: 10.3389/fbioe.2020.582953

\author{
Olanrewaju B. Morenikeji ${ }^{1 *}$, Kahleel Bernard ${ }^{1}$, Ellis Strutton ${ }^{1}$, Madeleine Wallace ${ }^{1}$ and \\ Bolaji N. Thomas ${ }^{2 *}$ \\ 'Department of Biology, Hamilton College, Clinton, NY, United States, ${ }^{2}$ Department of Biomedical Sciences, Rochester \\ Institute of Technology, Rochester, NY, United States
}

Coronavirus is a family of viruses including alpha-, beta-, gamma-, delta-coronaviruses. Only alpha- and betacoronaviruses have been observed to infect humans. Past outbreaks of SARS-CoV and MERS-CoV, both betacoronavirus, are the result of a spillover from animals. Recently, a new strain termed SARS-CoV-2 emerged in December 2019 in Wuhan, China. Severe cases of COVID-19, the disease caused by SARS-CoV-2, lead to acute respiratory distress syndrome (ARDS). One contributor to the development of ARDS is cytokine storm, an overwhelming inflammatory immune response. Long non-coding RNAs (IncRNAs) are genetic regulatory elements that, among many functions, alter gene expression and cellular processes. IncRNAs identified to be pertinent in COVID-19 cytokine storm have the potential to serve as disease markers or drug targets. This project aims to computationally identify conserved IncRNAs potentially regulating gene expression in cytokine storm during COVID-19. We found 22 IncRNAs that can target 10 cytokines overexpressed in COVID-19 cytokine storm, 8 of which targeted two or more cytokine storm cytokines. In particular, the IncRNA non-coding RNA activated by DNA damage (NORAD), targeted five out of the ten identified cytokine storm cytokines, and is evolutionarily conserved across multiple species. These IncRNAs are ideal candidates for further in vitro and in vivo analysis.

Keywords: IncRNA, cytokine storm, genes, markers, regulation, COVID-19

\section{INTRODUCTION}

Coronavirus is a family of respiratory viruses including alpha-, beta-, gamma-, and deltacoronaviruses, of which only alpha- and beta-coronaviruses have been observed to infect humans (Cui et al., 2019). Past outbreaks of SARS-CoV and MERS-CoV, both betacoronaviruses, are the result of zoonotic spillover (de Wit et al., 2016). In December 2019, a new strain of coronavirus termed SARS-CoV-2 emerged in a seafood market in Wuhan, China, thought to be a zoonotic spillover from pangolins (Sun et al., 2020; Zhang et al., 2020). While SARS-CoV-2 shares a $96 \%$ sequence similarity to a horseshoe bat coronavirus, there is ongoing research to identify a more recent intermediate host species (Mallapaty, 2020; Zhou P. et al., 2020). As of October 14, 2020, over 38.4 million cases of COVID-19, the disease caused by SARS-CoV-2, have been confirmed worldwide (Dong et al., 2020). Common symptoms of COVID-19 include fever, cough, pounding headaches, anosmia and 
ageusia, yet one unique attribute is the role of asymptomatic individuals in the spread of disease (Guan et al., 2020; Vaira et al., 2020). Asymptomatic spread is estimated to be responsible for $40-45 \%$ SARS-CoV-2 transmission, as compared to $9.8 \%$ in MERS-CoV (Al-Tawfiq, 2020; Oran and Topol, 2020). Severe cases of COVID-19 has been shown to lead to acute respiratory distress syndrome (ARDS), the leading cause of death from COVID-19 (Ruan et al., 2020). ARDS has a mortality rate of around 75\%; thus it is critical to develop a thorough understanding of this pathology, examine contributory factors, dissect possible role for host genetics in variable disease outcome and search for potential treatments (Yang et al., 2020).

One contributor to the development of ARDS is cytokine storm, a dysregulated, overwhelming pro-inflammatory immune response (Ye et al., 2020). Cytokines are the main mode of communication between innate immune cells, and are a part of a normal innate immune response that serves as the first line of defense against pathogens (Altan-Bonnet and Ratnadeep, 2019; Ye et al., 2020). However, an aggressive pro-inflammatory response can also circulate throughout the body and cause damage to tissue resulting in septic shock and multi-organ failure (Diao et al., 2020; Tay et al., 2020; Xiong et al., 2020). Multiple studies have identified cytokines upregulated in cytokine storm, including IL-2R, IL-6, IL-8, IL-10, and TNF $\alpha$ (Chu et al., 2020; Liu et al., 2020; Mehta et al., 2020; Qin et al., 2020). Thus, a potential treatment modality is the regulation of proinflammatory host immune response to COVID-19.

Currently, there are no approved antiviral treatments or vaccines specific for COVID-19 (Wu C. et al., 2020; Wu Y. et al., 2020). Current treatment consists of symptomatic and supportive care, with treatment of secondary conditions. Treatments currently in use include antivirals such as remdesivir and convalescent plasma (Chen G. et al., 2020; Chen L. et al., 2020; Chen N. et al., 2020; Chen X. et al., 2020). The current protocol for diagnosing COVID-19 is a viral RNA RT-PCR test with a nasopharyngeal or oropharyngeal sample (Beeching et al., 2020). Antibody tests using blood samples have also been developed to detect IgG and IgM antibodies indicative of a prior exposure. These tests are performed using ELISA and lateral flow immunoassays, and have the caveat of detecting cases after the first week of infection and lower sensitivity for asymptomatic individuals ( $\mathrm{Li}$ et al., 2020; Okba et al., 2020). A diversity of vaccine candidates are currently being tested, with 115 vaccine candidates and five vaccines in phase 1 clinical trials (Thanh Le et al., 2020). Concomitant with the need for treatments tailored to COVID-19 is the need for a diverse set of diagnostic and prognostic markers related to disease severity. Markers forecasting increased disease severity can serve as indicators for specific treatments before a severe disease phenotype is observed, leading to earlier interventions and better patient outcomes. So far, raised procalcitonin (PCT) levels and low platelet counts have been found to be associated with an increased risk of severe cases of COVID-19 infection and death (Li et al., 2020; Lippi and Plebani, 2020; Lippi et al., 2020). One avenue that is yet to be explored is COVID-19 diagnostic markers designed on the basis of gene regulation. While viral RNA load has been examined as a proxy for virus titer and a predictor of disease severity, no study to date has looked at endogenous genetic regulatory elements as markers of COVID-19 disease severity. Thus, this area warrants further inspection and characterization to inform treatment and diagnostic development.

Long non-coding RNAs, or lncRNAs, are non-coding RNA strands over 200 nucleotides in length that have structural, catalytic, or regulatory roles (Yang et al., 2014). So far, numerous lncRNAs including MALAT1, SNHG14, and XIST have been identified to play roles during inflammatory immune response (Chen C. C. et al., 2018; Chen H. et al., 2018; Zhong et al., 2018; Ma et al., 2019). LncRNAs NORAD, PAAN and NRON have been demonstrated to be important in modulating viral pathogenesis in hepatitis C, influenza A and HIV-1, respectively (Imam et al., 2015; Sur et al., 2018; Wang et al., 2018). Additionally, a database of differential lncRNA expressed in mice during SARSCoV pathogenesis through RNA-seq has been created (Josset et al., 2014), including a recent characterization of transcriptional lncRNA in normal bronchial epithelial cells, underscoring their importance in immune response regulation (Vishnubalaji et al., 2020). There has been significant progress made in the creation of tools to computationally identify and functionally annotate lncRNAs. Human ncRNA expression profiles have been created for normal tissues, cancer cell lines, and subcellular components (Djebali et al., 2012; Klijn et al., 2015; Uhlén et al., 2015). Functionally, softwares are available to predict lncRNA-RNA and lncRNA-protein interactions (Kato et al., 2010; Bellucci et al., 2011; Kiryu et al., 2011; Lu et al., 2013). Tools are also available to predict lncRNA secondary structures, consensus secondary structures, tertiary structures and joint secondary structures, and a database has also been created to compile lncRNA-target relationships from literature (Iwakiri et al., 2016; Cheng et al., 2019). However, the lncRNA transcriptome has yet to be comprehensively annotated; challenges in the area of lncRNA research include the relative low expression levels of lncRNAs, lack of understanding of the lncRNA sequencefunction relationship, and weak conservation during evolution (Uszczynska-Ratajczak et al., 2018). Using publicly available lncRNA databases and computational tools, we sought to identify lncRNAs involved in COVID-19 cytokine storm and understand their role in disease pathology.

\section{MATERIALS AND METHODS}

\section{Identification of Significant Cytokines in COVID-19 Cytokine Storm}

Cytokines associated with increased COVID-19 cytokine storm were identified using the search engines Google Scholar, PubMed, and Web of Science, and a literature review by Costela-Ruiz et al. (2020) amongst others. The literatures were retrieved and manually curated for cytokine reports in several COVID-19 patient cases. Our literature search included only publications between 2019 and 2020 capturing the period of COVID-19 outbreak. Based on the reports from published literatures, cytokines selected for further analysis were corroborated by at least 3 sources to be associated with increased COVID-19 severity and subsequent cytokine storm. 


\section{Prediction of Cytokine-IncRNA Relationships}

Cytokines identified in the previous step were queried in the LncRNA2Target, a database compiling IncRNA-target relationship, as described by Cheng et al. (2019). Briefly, cytokine official gene names were used to query against human lncRNAs database, using default settings. Sequences for identified lncRNAs were retrieved from NONCODE (noncode.org) (Zhao et al., 2016), NCBI Gene (ncbi.nlm.nih.gov/gene), and NCBI Gene Expression Omnibus (GEO) (ncbi.nlm.nih.gov/geo). In order to ascertain the binding ability of IncRNA-target interactions, we assessed a minimum free energy for each cytokine-lncRNA gene pair previously identified by LncRNA2Target. LncTar was used to calculate the normalized binding free energy (ndG) for lncRNAs and their associated cytokine genes as described (Li et al., 2015). LncRNA sequences are usually long, therefore, where too long sequences were found, to be accepted by LncTar were divided into sections around $17,500 \mathrm{nt}$ in length to be run individually.

\section{Protein-Protein Network Analysis of Significant Cytokines in COVID-19 Cytokine Storm}

To gain insight into molecular interactions between cytokines and pathogenic mechanisms, we performed a protein-protein interaction network analysis of the 10 most significant cytokines using Search Tool for the Retrieval of Interacting Genes database (STRING-DB;string-db.org, Szklarczyk et al., 2017). The gene sequences associated with the official gene names were retrieved from ensembl database (ensembl.org) and used in performing the network analysis as described (Jiang et al., 2019; Morenikeji and Thomas, 2019). In order to visualize the functional relationships between IncRNAs, cytokines targets, and metabolic pathways, a network analysis was created using Cytoscape (v3.7.2) (cytoscape.org). To identify significant nodes in the network, Molecular Complex Detection (MCODE); a Cytoscape plug-in was used to generate network clustering based on regions with dense connections (Jiang et al., 2019). We hypothesize that lncRNA that target multiple genes with the same molecular function will perturb or regulate the same pathways during the disease. The lncRNA-target-pathways network was constructed with Cytoscape (v3.7.2) program (Smoot et al., 2011).

\section{Pathway Enrichment, Functional Annotation, and Gene Ontology Analysis of Significant Cytokines and Identified IncRNAs Associated With COVID-19 Cytokine Storm}

In order to elucidate pathways that are significantly perturbed in COVID-19 cytokine storm, GeneAnalytics (geneanalytics.genecards.org) was used to identify metabolic pathways and gene ontology terms associated with such cytokines. Likewise, to understand the role that the identified lncRNAs play in disease pathology, we used GeneAnalytics to identify associated diseases, as described (Fuchs et al.,
2016). Metabolic pathways, gene ontology terms, and diseases selected for further analysis had a corrected $p$-value equal to or below 0.0001 and were associated with 4 or more cytokines. GeneAnalytics was used to identify diseases associated with the lncRNAs identified previously. Diseases selected for further analysis had a corrected $p$-value equal to or below 0.0001 and were associated with 4 or more lncRNAs.

\section{Evolutionary Trace of Non-coding RNA Activated by DNA Damage (NORAD), Structural Prediction and Characterization}

The lncRNA NORAD (Non-Coding RNA Activated by DNA Damage) found from the previous steps was significantly predicted/reported to target more cytokine genes than any other lncRNA, so it was selected for further evolutionary trace analysis into to depict its evolutionary conservation among other species. Although, IncRNAs are known to be poorly conserved, therefore we proposed that a lncRNA targeting multiple genes with high evolutionary conservation would be a significant maker during COVID-19 cytokine storm. NCBI BLASTn was used to search for related lncRNAs in other species against the NORAD nucleotide sequence. Out of the top 100 results returned, one sequence was selected for each genus returned. A multiple sequence alignment and phylogenetic tree was created using MEGA (v7), with the neighbor-joining clustering method and bootstrapping with 500 iterations (Kumar et al., 2016). The phylogenetic tree was imported to ITOL (itol.embl.de) for proper visualization (Letunic and Bork, 2019). In addition, based on the annotated human Reference Sequence (RefSeq) of NORAD (LINC00657), we obtained its mammalian conservation across hundreds of genomic sequences and expressed sequence tags (ESTs) using UCSC Genome Browser (https://genome.ucsc.edu/cgi-bin/hgGateway). In order to gain insight into the thermodynamic stability and evolutionary conservation of lncRNA NORAD secondary structure, we carried out a multiple sequence alignment of six mammals (human, chimpanzee, monkey, rat, mouse and dog) and transferred to RNAzWebServer (http://rna.tbi.univie.ac.at/cgi-bin/RNAz/ RNAz.cgi), using default parameter as described (Mathews et al., 2004; Lorenz et al., 2011). This server uses dynamic programming algorithm to determine encoding base-pair probabilities and RNA folding. A predicted RNA structure from the MSA with probability higher than $0.5(p>0.5)$ is considered a strong evidence for structural RNA and evolutionary conservation.

\section{RESULTS}

\section{Identification of Significant Cytokines in COVID-19 Cytokine Storm}

As of June 3, 2020, 210 papers appear in Web of Science, 518 papers in PubMed, and 6,420 results in Google Scholar for COVID-19 cytokine storm. Our literature search revealed a total of 17 papers that identified 28 cytokines to be significantly involved or associated with COVID-19 cytokine storm (Figure 1A, Supplementary Table 1). To pin-point significantly reported cytokines, ten cytokines met the threshold for 

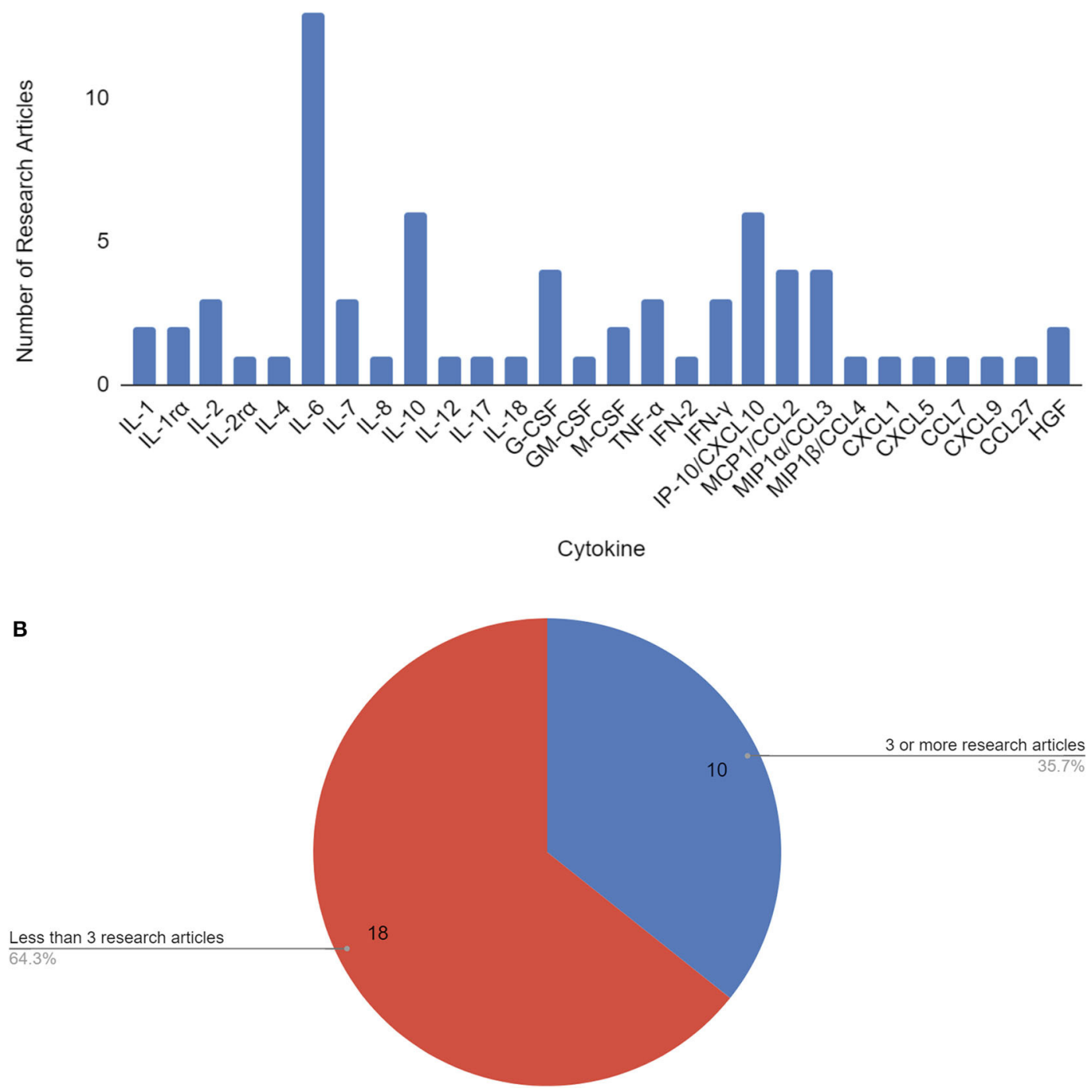

FIGURE 1 | Cytokines by number of research articles identifying the cytokine to be associated with COVID-19 disease severity and/or cytokine storm (A). Cytokines used in further analysis (10) were associated with 3 or more papers that cited them as correlated with COVID-19 disease severity and/or cytokine storm (B).

consideration of having 3 or more research articles citing it as being associated with COVID-19 disease severity and/or cytokine storm, and were selected for further analysis (Table 1, Figure 1B). IL-6, IL-10, and IP-10 were mentioned most often in literature as being significant, being mentioned in 13, 6 , and 6 papers, respectively.

\section{Prediction of Cytokine-IncRNA Relationships}

We identified a total of 24 cytokine-lncRNA relationships using LncRNA2Target (Table 2). Two lncRNAs were removed from further analysis due to identical primer sequences. Thus, 22 lncRNAs were selected for further analysis. All lncRNA-target pairs identified by LncRNA2Target had a normalized binding free energy (ndG) above -0.02 (Supplementary Table 2). The lncRNAs LNC-LBCS, STXBP5-AS1, and CDK6-AS1 were too long to run in LncTar and had to be divided into multiple sections for analysis. The lncRNA NORAD was found to pair with five out of ten of the cytokines in our study including IL-6, IL-10, CSF3, TNF $\alpha$ and CXCL10, more than any other lncRNAs (Table 3). Therefore, this lncRNA was selected for further evolutionary trace analysis to identify its conservation across other species.

\section{Protein-Protein Network Analysis of Significant Cytokines in COVID-19 Cytokine Storm}

A protein-protein network of the 10 most significant cytokines in COVID-19 cytokine storm was created to elucidate molecular 
TABLE 1 | COVID-19 cytokine storm significant cytokines used for further IncRNA analysis.

\begin{tabular}{|c|c|c|}
\hline Cytokine target & Official gene name & mRNA sequence accession number \\
\hline IL-2 & IL-2 & BC070338.1 \\
\hline IL-6 & IL-6 & BC015511.1 \\
\hline IL-7 & IL-7 & BC047698.1 \\
\hline IL-10 & IL-10 & BC104253.1 \\
\hline G-CSF & CSF3 & ВС033245.1 \\
\hline TNF $\alpha$ & TNF $\alpha$ & BC028148.1 \\
\hline $\mathrm{IFN} \gamma$ & $\mathrm{IFN} \gamma$ & BC070256.1 \\
\hline IP-10/CXCL10 & CXCL10 & BC010954.1 \\
\hline MCP1/CCL2 & CCL2 & BC009716.1 \\
\hline MIP1a/CCL3 & CCL3 & BC071834.1 \\
\hline
\end{tabular}

Official gene names and mRNA sequence accession number are shown.

TABLE 2 | The most significant IncRNAs associated with COVID-19 cytokine storm cytokines, accompanied by IncRNA target prediction analysis using average ndG values.

\begin{tabular}{|c|c|c|c|}
\hline Cytokine target & $\begin{array}{l}\text { Number of } \\
\text { IncRNAs }\end{array}$ & IncRNA gene names & Average ndG \\
\hline $\mathrm{IL}-2$ & 3 & $\begin{array}{l}\text { BANCR, InrCXCR4, } \\
\text { DRAIC }\end{array}$ & -0.0392 \\
\hline IL-6 & 7 & $\begin{array}{l}\text { Inc-IL7R }{ }^{\star} \text {, LNCSRLR, } \\
\text { SBF2-AS1, } \\
\text { RAD51-AS1, } \\
\text { LNC-LBCS, NORAD, } \\
\text { SENCR }\end{array}$ & -0.038409091 \\
\hline $\mathrm{IL}-7$ & 2 & TUG1, SBF2-AS1 & -0.06735 \\
\hline $\mathrm{IL}-10$ & 4 & $\begin{array}{l}\text { GAS5, InrCXCR4, } \\
\text { NORAD, SNHG1 }\end{array}$ & -0.0669 \\
\hline CSF3 & 2 & STXBP5-AS1, NORAD & -0.045418182 \\
\hline TNF $\alpha$ & 6 & $\begin{array}{l}\text { THRIL, RAD51-AS1, } \\
\text { CASC15, NORAD, } \\
\text { GAS5, NRCP* }\end{array}$ & -0.044842857 \\
\hline $\mathrm{IFN} \gamma$ & 2 & TMEVPG1, PRC1-AS1 & -0.0257 \\
\hline CXCL10 & 1 & NORAD & -0.0319 \\
\hline CCL2 & 5 & $\begin{array}{l}\text { MALAT1, TUG1, } \\
\text { RAD51-AS1, SNHG1, } \\
\text { NRAV }\end{array}$ & -0.04588 \\
\hline CCL3 & 3 & $\begin{array}{l}\text { InrCXCR4, NRAV, } \\
\text { CDK6-AS1 }\end{array}$ & -0.067471429 \\
\hline
\end{tabular}

InCRNAs with an asterisk are not official gene names, and are not annotated in NCBI gene. ndG; a cutoff to determine whether an RNA molecule interacts with target gene.

interaction and possible mechanism for co-expression using STRING-DB (Figure 2). We found IL-2 connected to the other cytokines only through text mining, while all other cytokines are interconnected with each other through both text mining and co-expression. In addition, IL-6, TNF $\alpha$, CCL3, CXCL10, and IFN $\gamma$ are all connected to IL-10 through interactions identified from curated databases. TNF is experimentally determined to be connected to IFN $\gamma$. A network analysis of lncRNA and cytokine pathway associations was created using Cytoscape (Figure 3). The lncRNAs lnrCRCX4, NORAD, and RAD51-AS1 significantly
TABLE 3 | The most significant IncRNAs associated with COVID-19 cytokine storm cytokines, along with the IncRNA accession number.

\begin{tabular}{|c|c|c|}
\hline IncRNA gene name & $\begin{array}{c}\text { Number of } \\
\text { cytokine targets }\end{array}$ & Accession number \\
\hline NORAD & 5 & NONHSAT079548.2 \\
\hline RAD51-AS1 & 3 & NONHSAT041865.2 \\
\hline InrCXCR4 & 3 & GSE104018 \\
\hline SBF2-AS1 & 2 & NONHSAT017939.2 \\
\hline TUG1 & 2 & NONHSAT084833.2 \\
\hline GAS5 & 2 & $\begin{array}{l}\text { NC_000001.11:c173868882- } \\
173863899\end{array}$ \\
\hline SNHG1 & 2 & NONHSAT021826.2 \\
\hline NRAV & 2 & NONHSAT031176.2 \\
\hline BANCR & 1 & NONHSAT131775.2 \\
\hline DRAIC & 1 & $\begin{array}{l}\text { NC_000015.10:69561720- } \\
69571440\end{array}$ \\
\hline Inc-IL7R ${ }^{\star}$ & 1 & AL713738.1 \\
\hline LNCSRLR & 1 & $\begin{array}{l}\text { NC_000003.12:c146069185- } \\
146066344\end{array}$ \\
\hline LNC-LBCS & 1 & $\begin{array}{l}\text { NC_000006.12:c19804759- } \\
19729421\end{array}$ \\
\hline SENCR & 1 & NONHSAT025072.2 \\
\hline STXBP5-AS1 & 1 & $\begin{array}{l}\text { NC_000006.12:C147204614- } \\
146841388\end{array}$ \\
\hline THRIL & 1 & NONHSAT164169.1 \\
\hline $\mathrm{NRCP}^{*}$ & 1 & NR_046371.2 \\
\hline TMEVPG1 & 1 & NONHSAT029277.2 \\
\hline PRC1-AS1 & 1 & $\begin{array}{l}\text { NC_000015.10:90966369- } \\
\text { 90988624 }\end{array}$ \\
\hline MALAT1 & 1 & $\begin{array}{l}\text { NC_000011.10:65497738- } \\
65506516\end{array}$ \\
\hline CDK6-AS1 & 1 & $\begin{array}{l}\text { NC_000007.14:92836483- } \\
\text { 92917187 }\end{array}$ \\
\hline CASC15 & 1 & NONHSAT108049.2 \\
\hline
\end{tabular}

InCRNAs with an asterisk are not official gene names; not annotated in NCBI database.

target or regulate 3 or more cytokines, while GAS5, NRAV, TUG1, SBF2-AS1, and lincIRX5 lncRNAs regulate two cytokines. Three major groups of pathways were cataloged based on the number of cytokines present in the pathway; in particular, it was found that eight pathways were associated with the cytokine IL-6.

\section{Pathway Enrichment, Functional Annotation, and Gene Ontology Analysis of Significant Cytokines and Identified IncRNAs Associated With COVID-19 Cytokine Storm}

GeneAnalytics mapped 10 out of 10 significant cytokines and 19 out of 21 lncRNAs with official gene names. Forty eight pathways were found to be associated with the 10 cytokines, including numerous molecular, cellular and disease pathways (Figure 4, Supplementary Table 3). All of the significant cytokines were involved in three pathways: PEDF induced signaling, cytokine signaling in immune system, and innate immune system. All 


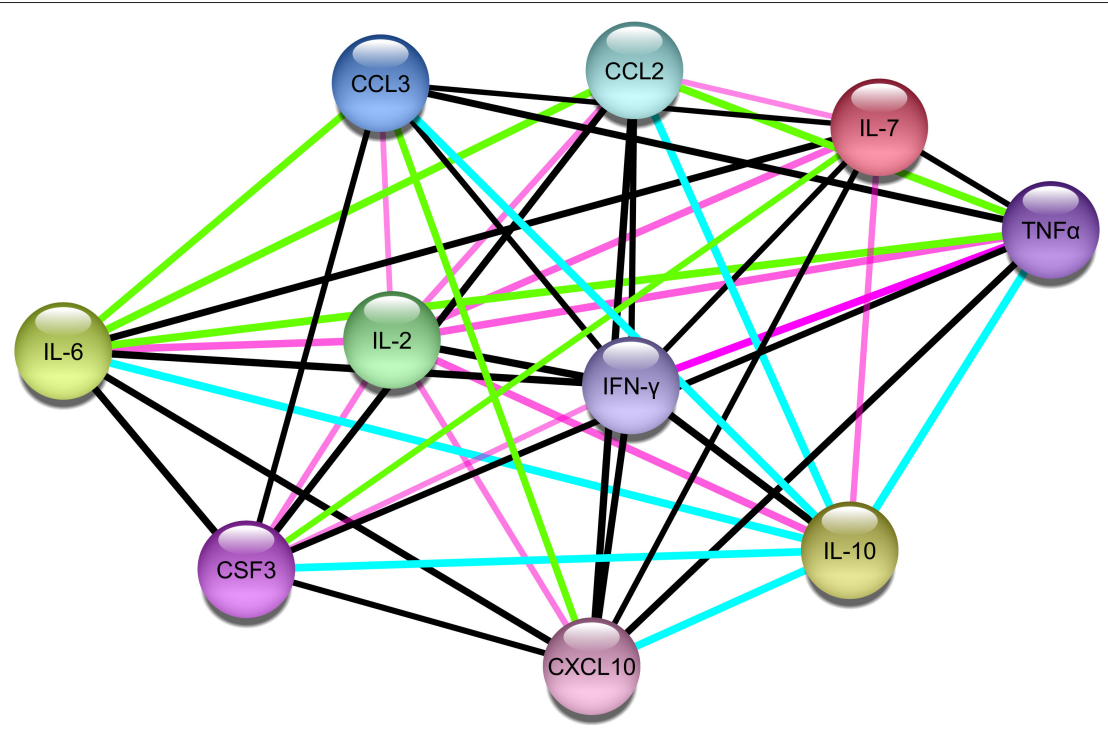

FIGURE 2 | Protein-protein interaction network of the 10 cytokines associated with COVID-19 cytokine storm (Edge color legend; blue: from curated database; pink: experimentally determined; green: text mining; black: co-expression).

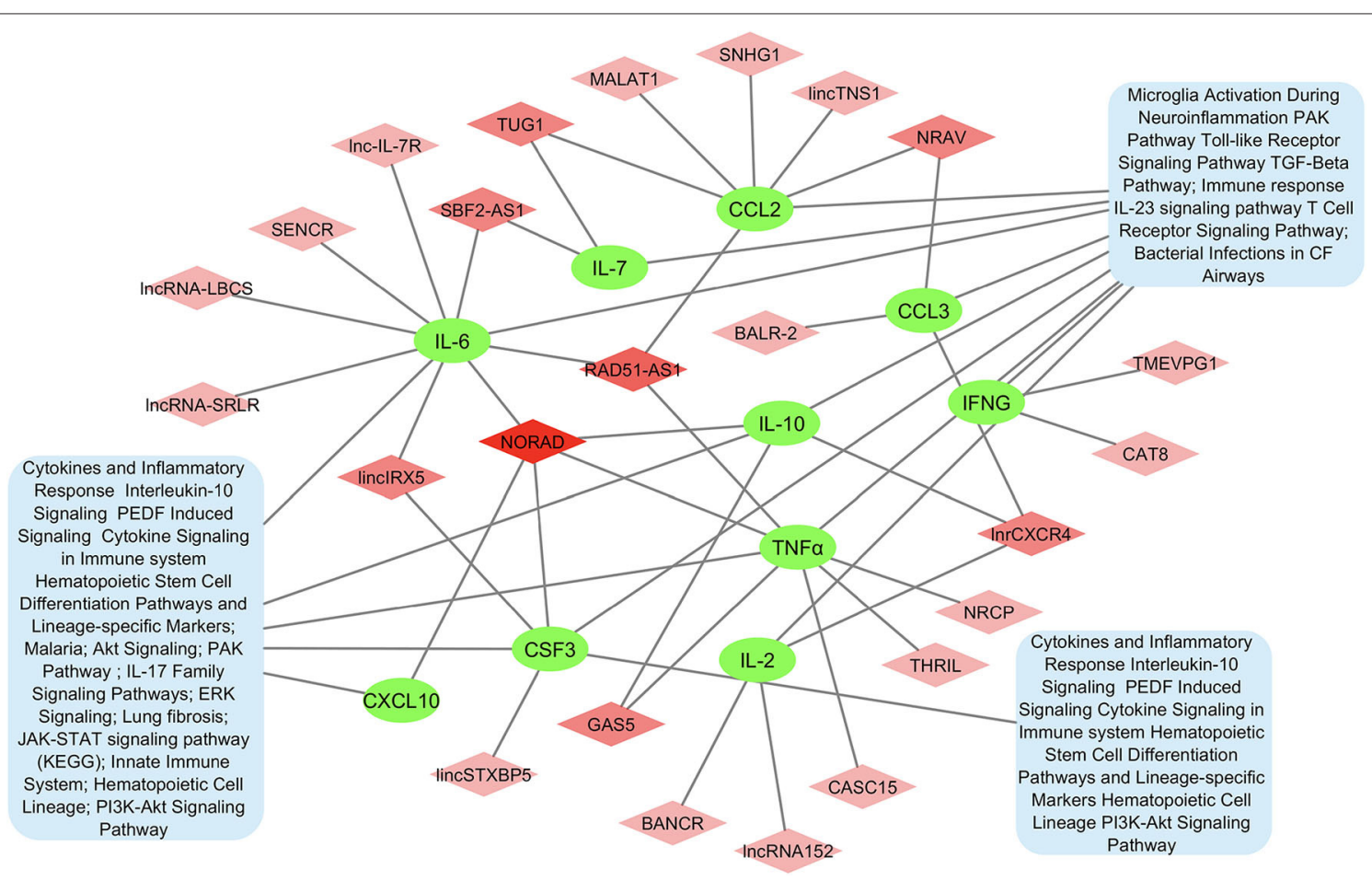

FIGURE 3 | Network of IncRNA and cytokine pathway associations for most significant cytokines in COVID-19 cytokine storm. Cytokines are shown in green, IncRNAs associated with multiple cytokines are shown in darker shades of pink, and pathways are shown in blue.

significant cytokines except for IFN $\gamma$ are also involved in the pathways: Akt signaling, PAK pathway, and ERK signaling.

Fifteen biological processes, 2 cellular components and 1 molecular function gene ontology terms were identified to be associated with the 10 significant cytokines (Table 4). All of the significant cytokines correlated with four gene ontology terms: cytokine activity, immune response, extracellular space, and extracellular region. One biological process, cytokinemediated signaling pathway, is correlated with all significant cytokines except for IFN $\gamma$. Twenty cancer related diseases 


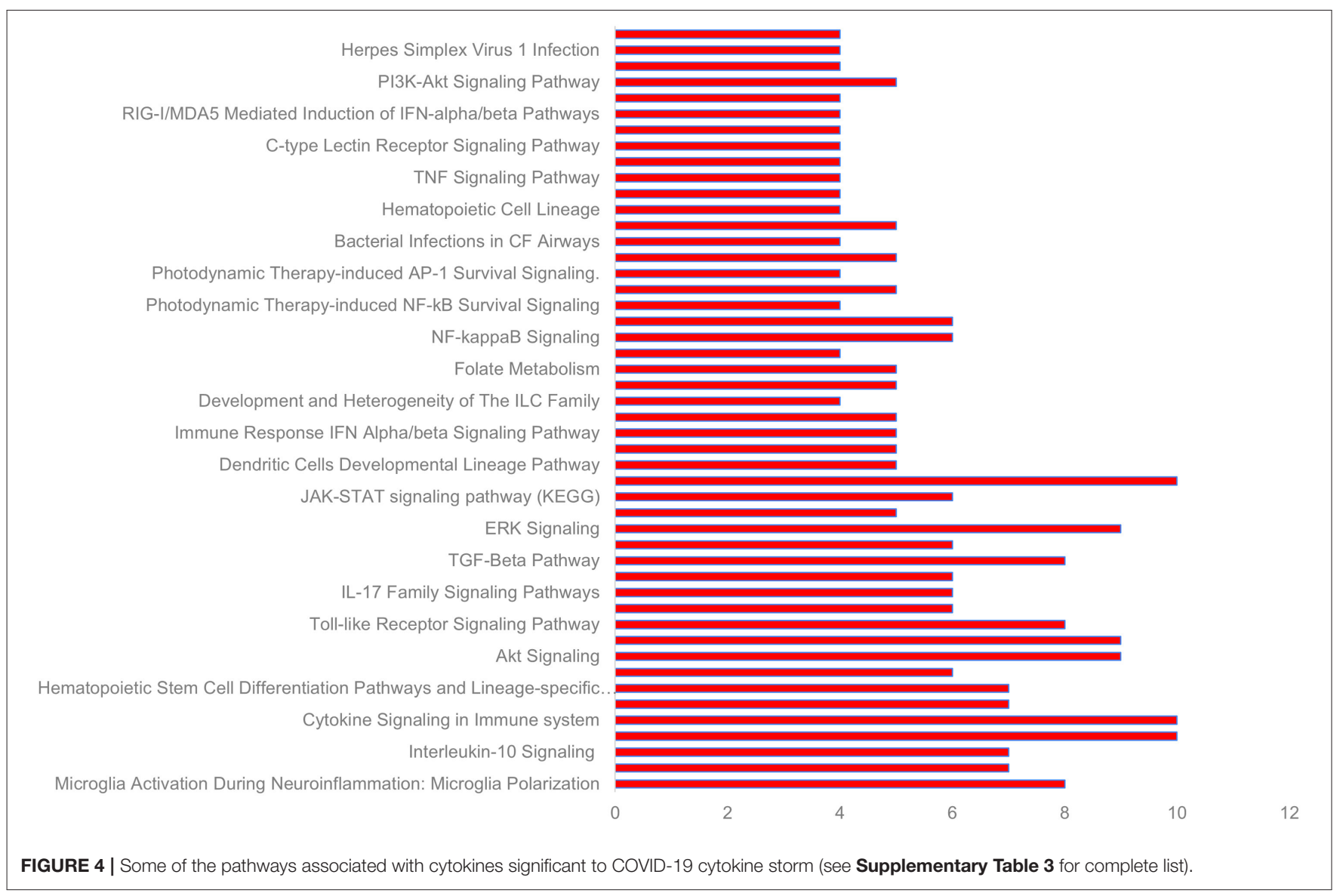

were found to be associated with the identified lncRNAs (Table 5), The cancers identified are not localized, and span across multiple organ systems. The disease with the most hits, hepatocellular carcinoma, is associated with ten out of nineteen mapped lncRNAs.

\section{Evolutionary Trace of IncRNA NORAD and Stable Folding}

Twenty three unique genera were represented in the top 100 results from a NCBI BLASTn search using the lncRNA NORAD. For genera with multiple species represented in the results, the species with the max score was selected for phylogenetic analysis. A phylogenetic tree, labeled by species and accession number was created and visualized (Figure 5). There are three major ingroups, though two of the three ingroups have moderate bootstrap values $(0.7$ and 0.654$)$. The ingroup with the Homo sapiens NORAD sequence consists entirely of primates. The Homo sapiens NORAD sequence was most phylogenetically related to another lncRNA found in Pan paniscus, with a bootstrap value of 1 . The 23 sequences represented in the tree are not exclusively from primates; 6 of the 9 sequences in the ingroup shown in black are from non-primate species. Figure 6A depicts annotated NORAD conserved regions across several mammalian genomic sequences and ESTs from the UCSC genome browser, while Figures 6B-E show two representation of significantly conserved RNA folding and multiple sequence alignment among six mammals (human, chimpanzee, monkey, rat, mouse and dog) with $p$-values of 0.92 and 1.0 , respectively. A $p$-value above 0.5 shows that lncRNA NORAD has a significantly high evolutionary conservation among these mammalian genomes and that the higher folding strength of NORAD is above threshold, an additional evidence of evolutionary conservation.

\section{DISCUSSION}

SARS-CoV-2 is a novel pathogen that has evolved into a pandemic, with significant mortality and morbidity rates. With millions infected, it is imperative to develop a better understanding of its pathology in order to develop treatments. An indicator for increased COVID-19 severity is cytokine storm, of which its genetic regulatory elements are poorly understood. We identified lncRNAs that can target significant cytokines during COVID-19 cytokine storm with computational tools. Ten cytokines significant in COVID-19 cytokine storm were selected out of a review of 17 papers for further analysis (as of May 2020). The cytokine IL-6 was identified to be significant in 13 out of 17 papers reviewed. IL-6 is currently being investigated as a potential drug target, with clinical trials underway for the IL-6 antagonist tocilizumab (Guaraldi et al., 2020; Kewan et al., 2020; Liu et al., 2020; Xu et al., 
TABLE 4 | Gene ontology terms associated with cytokines significant to COVID-19 cytokine storm.

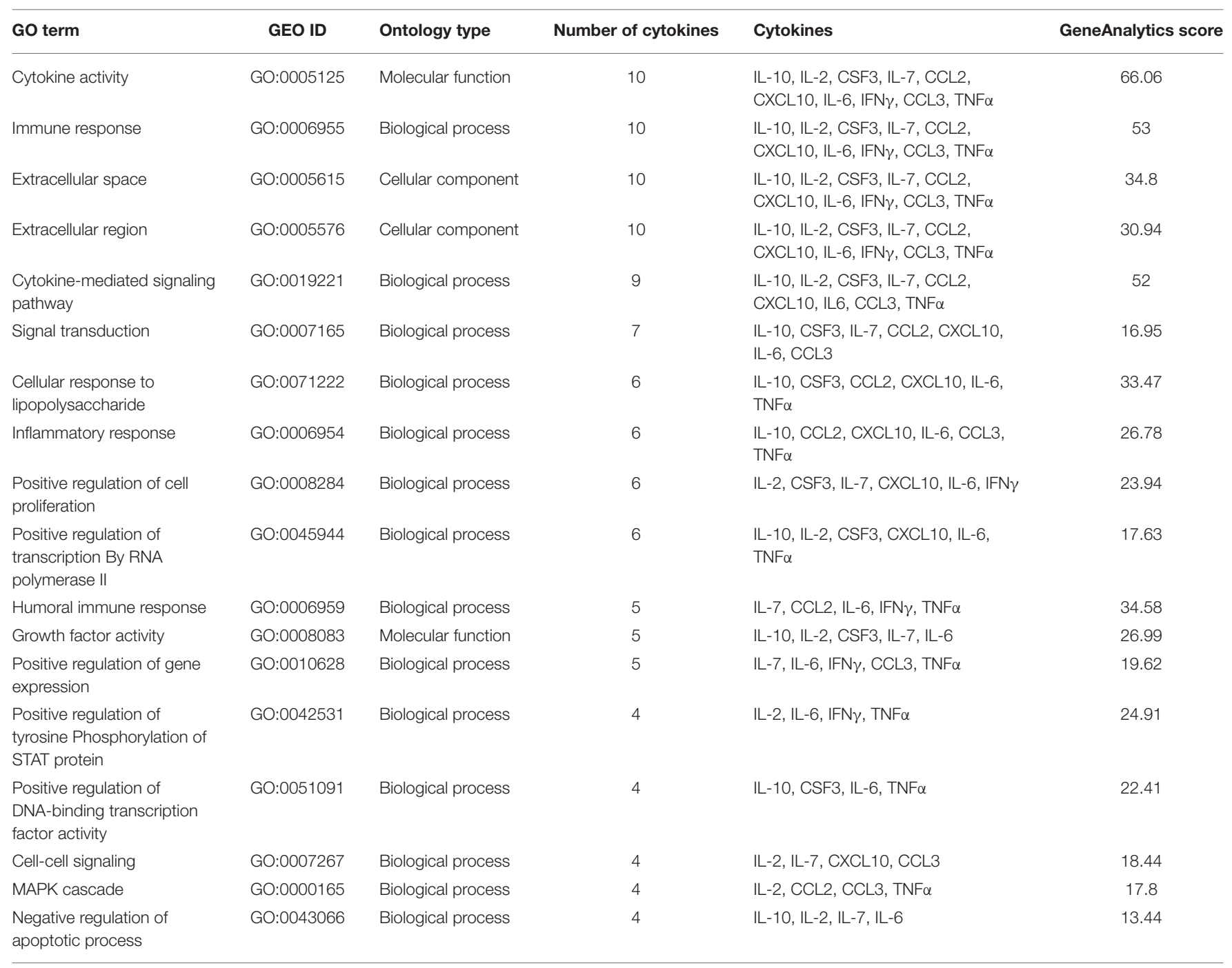

2020). A total of $22 \operatorname{lncRNAs}$ were identified to bind with the 10 significant cytokines, all with binding free energies <0.02 . The lncRNA NORAD targeted 5 of the 10 significant cytokines, while the lncRNAs RAD51-AS1 and lnrCXCR4 each target three of the significant cytokines. Since lncRNAs are known to contribute to transcriptional and epigenetic regulation as well as post transcriptional modifications, we hypothesize that $\operatorname{lncRNAs}$ that are able to target and bind to significant cytokine nucleotide sequences have the potential to downregulate cytokine expression, which can ameliorate the pro-inflammatory immune response to COVID-19 infection, mitigating cytokine storm in the process. Potential translational approaches to administering this technology in the clinical setting include antisense oligonucleotides knockdown, RNAi knockdown, and viral gene therapy (Fatemi et al., 2014; Lennox and Behlke, 2016; Roberts et al., 2020).

NORAD targeted five of the ten cytokines involved in cytokine storm, more than any other identified lncRNA.
NORAD, short for non-coding RNA activated by DNA damage, is responsible for chromosome stability and mitotic division (Lee et al., 2016; Tichon et al., 2016). Upregulation of NORAD is associated with 6 different types of cancers, and overexpression of NORAD leads to poor overall survival in cancer patients (Yang et al., 2019). In the context of SARS-CoV-2 viral infection, upregulation of NORAD may be a response to aberrant viral nucleotide replication within macrophages.

RAD51-AS1 is shown to inhibit DNA repair, and has a conserved E2F1 binding site in its promoter region (Zhang et al., 2017). RAD51-AS1 has been shown to inhibit DNA damage repair ability in hepatocellular carcinoma cells. E2F1 is a transcription factor that regulates the cell cycle and apoptosis (Qin et al., 1994; Shan and Lee, 1994; Wu and Levine, 1994). RAS51-AS1 may be expressed in the context of viral replication within macrophages. RAD51-AS1 has also been proposed as a prognostic marker for epithelial ovarian cancer (Zhang et al., 
TABLE 5 | Diseases associated with identified IncRNAs in COVID-19 cytokine storm.

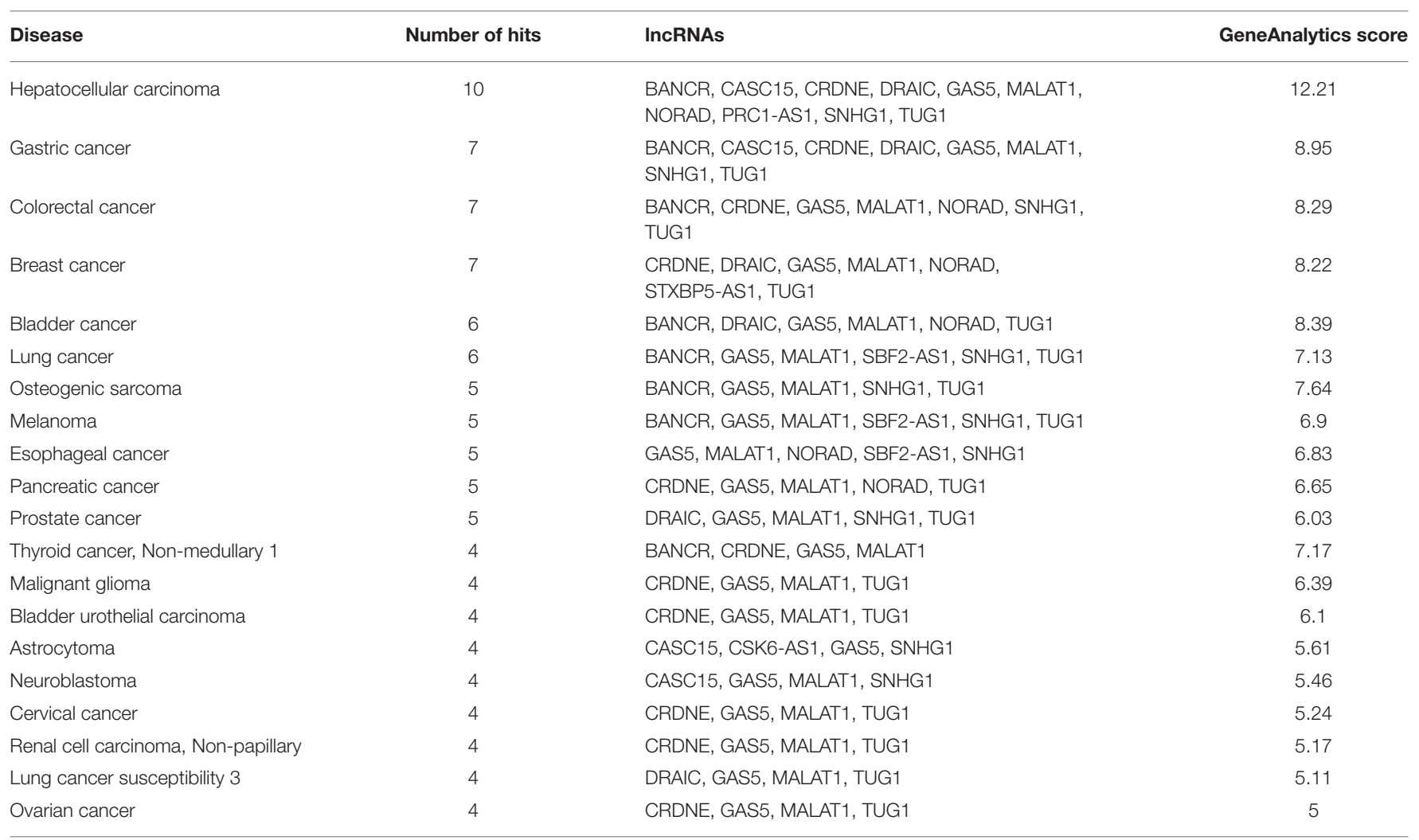

2017). In the context of SARS-CoV-2 infection, RAD51-AS1 may be expressed in response to cellular damage from viral replication within macrophages, leading to expression of pro-inflammatory cytokines. The lncRNA lnrCRCX4 has an NCBI GEO entry, but does not have any associated published literature.

The lncRNAs SBF2-AS1, TUG1, GAS5, SNHG1, and NRAV all target two of the significant cytokines. SBF2-AS1 has been proposed as a disease marker for A. fumigatus (Riege et al., 2017). TUG1 knockdown has been found to decrease inflammatory response in atherosclerotic lesions (Zhang $H$. et al., 2018). In contrast, TUG1 overexpression results in decreased levels of pro-apoptotic factors and inflammation in lipopolysaccharide exposed H9c2 cells cytokines (Zhang L. et al., 2018). GAS5 has also been shown to inhibit NF- $\mathrm{B}$ and Notch signaling pathways and reduce lipopolysaccharide inflammatory injury in ATDC5 chondrocytes (Li et al., 2018). SNHG1 upregulation has been found to significantly decrease the production of pro-inflammatory cytokines-NO, PGE2, IL-6, and TNF $\alpha$ in human chondrocytes (Lei et al., 2019). These lncRNAs have been demonstrated to have the potential to be disease markers and negative regulators of pro-inflammatory cytokines.

Notably NRAV, abbreviated for negative regulation of antiviral response, is shown to downregulate interferon stimulating genes (ISG) (Ouyang et al., 2014). Silencing NRAV has been shown to suppress influenza A virus replication and virulence. Interestingly, the receptor for COVID-19, ACE2, is an ISG (Ziegler et al., 2020). Because SARS-CoV-2 must balance aggravating the host immune response and promoting infectivity, the expression levels of NRAV would be an interesting lncRNA for future studies to characterize gene expression.

The metabolic pathways that involve 8 or more of the 10 significant cytokines; PEDF signaling, cytokine signaling in the immune system, innate immune system, Akt signaling, PAK pathway, and ERK signaling; are all related to an inflammatory response to a pathogen (Kurosawa et al., 2000; Yabe et al., 2005; Chan et al., 2007; Sun et al., 2018). Likewise, the gene ontology terms associated with the 10 significant cytokines are consistent with pro-inflammatory innate immune response. The diseases associated with the identified lncRNAs are cancers of multiple organ systems possibly because lncRNAs are tissue specific. The breadth of the organ systems affected may be linked to the systemic nature of cytokine storms. Cancers are a dysregulation of normal cellular processes resulting in uncontrolled growth; thus, it parallels with cytokine storm in that both are hyperactivations of normal cellular processes.

An evolutionary trace was performed on the lncRNA NORAD using 23 highly similar sequences from neighboring species. Despite lncRNAs as a whole being poorly conserved (Hezroni et al., 2015), NORAD shows a high degree of conservation among related species, especially in mammals. All of the sequences in the same branch of the tree (in blue) as the Homo sapiens NORAD sequence are primates. The species sharing the closest common ancestor, Pan paniscus, is paired with the Homo sapiens sequence with a bootstrap value of 1 . This indicates that Pan paniscus 


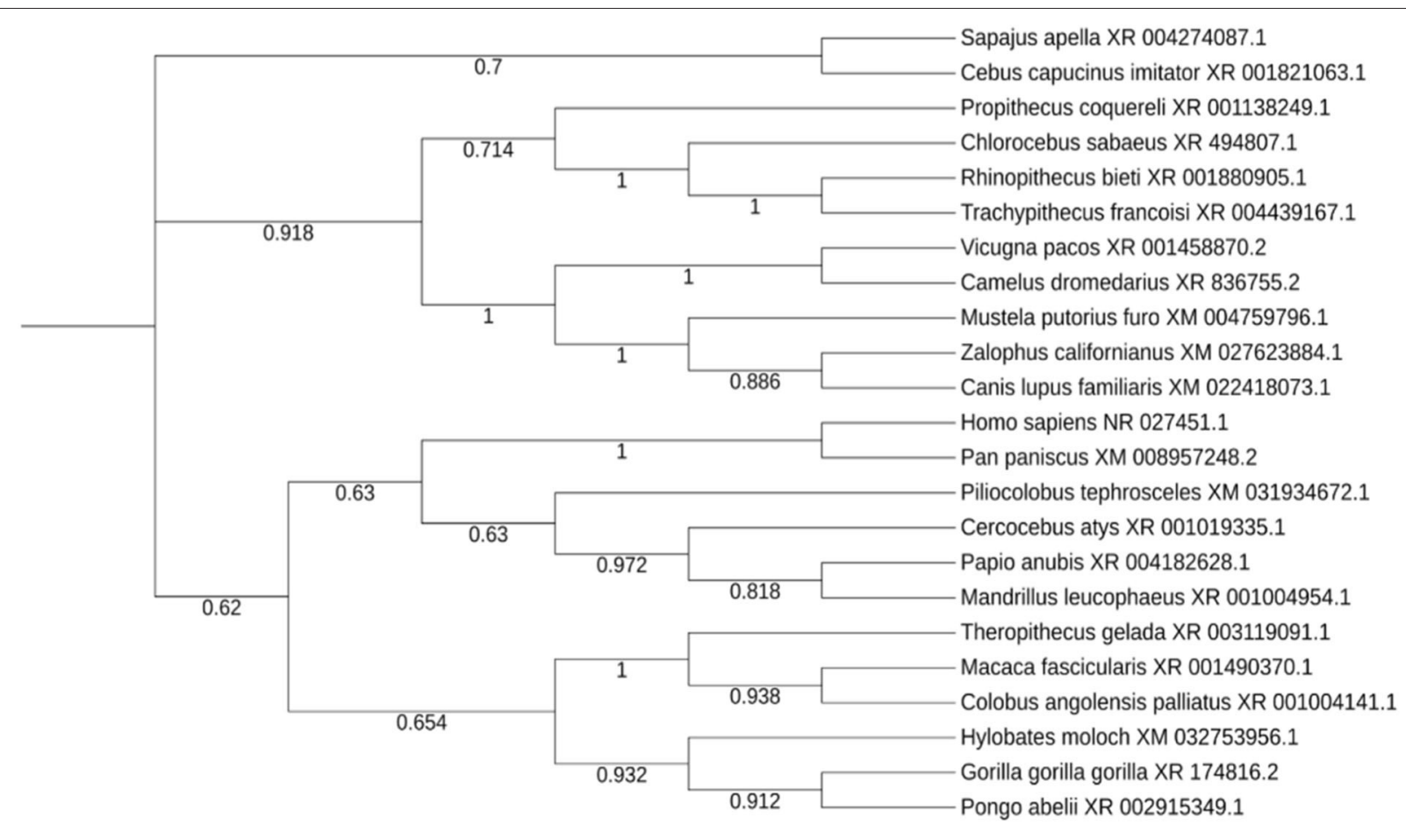

FIGURE 5 | Evolutionary trace of IncRNA NORAD in 23 related species, with bootstrap values at each node.

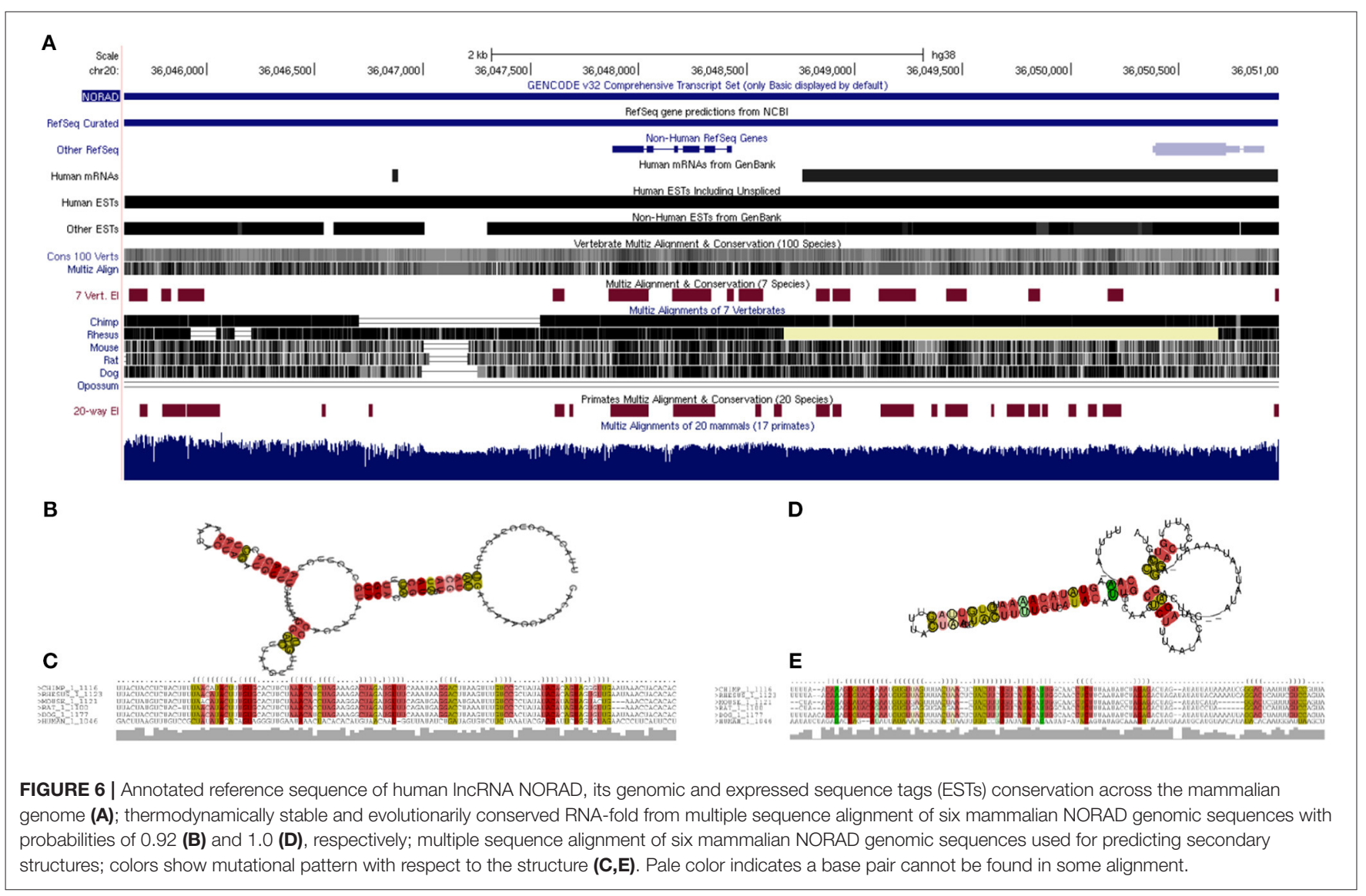


is a promising model organism for further evaluating the role and pathology of NORAD in COVID-19 disease pathology, and potentially useful for testing new medications before clinical trials in humans. LncRNAs could fold in diverse complex manner to form secondary and varying functions. Our study reveal a strong evidence of conserved NORAD structural folding across mammalian genomes with a high thermodynamic stability, given its peculiar ability to interact or bind many cytokines. In addition, its high folding strength suggests a positive correlation with expression and functional significance. Taken together, these results provide evidence of significant evolutionary conservation and functional stability across mammalian genomes, further strengthening its candidacy for gene regulation during immune response to SARS-CoV-2 perturbation.

\section{CONCLUSION}

In SARS-CoV-2 infection, no treatments are approved to treat cytokine storm, a precursor to ARDS. We sought to computationally identify cytokines that were significantly upregulated in COVID-19 cytokine storm, and lncRNAs that can target these cytokines. From literature review, we found 10 cytokines to be significantly upregulated in COVID-19 cytokine storm and were selected for further analysis. We identified 22 lncRNAs that can target these cytokines, 8 of which can target multiple cytokines. Of particular and possibly clinical importance, we report that lncRNA NORAD can target 5 of the 10 significant cytokines. Though lncRNAs are known to be less conserved across species, conversely and of interest, we found NORAD to be highly conserved across multiple mammalian species, in addition to previous reports of its overexpression associated with multiple cancer phenotypes. Additionally, 5 lncRNAs that target multiple cytokines have been experimentally identified to have roles in inflammatory responses. Therefore, these lncRNAs show potential as targets for intervention during SARS-CoV-2 pathogenesis, and are prime candidates for further in vivo and in vitro analysis.

\section{REFERENCES}

Altan-Bonnet, G., and Ratnadeep, M. (2019). Cytokine-mediated communication: a quantitative appraisal of immune complexity. Nat. Rev. Immunol. 19, 205-217. doi: 10.1038/s41577-019-0131-x

Al-Tawfiq, J. A. (2020). Asymptomatic coronavirus infection: MERS-CoV and SARS-CoV-2 (COVID-19). Travel Med. Infect. Dis. 35:101608. doi: 10.1016/j.tmaid.2020.101608

Beeching, N. J., Fletcher, T. E., and Beadsworth, M. B. J. (2020). Covid-19: testing times. BMJ 369:m1403. doi: 10.1136/bmj.m1403

Bellucci, M., Agostini, F., Masin, M., and Tartaglia, G. G. (2011). Predicting protein associations with long noncoding RNAs. Nat. Methods 8, 444-445. doi: 10.1038/nmeth.1611

Chan, C. M., Ma, C. W., Chan, W. Y., and Chan, H. Y. E. (2007). The SARScoronavirus membrane protein induces apoptosis through modulating the Akt survival pathway. Arch. Biochem. Biophys. 459, 197-207. doi: 10.1016/j.abb.2007.01.012

Chen, C. C., Chen, C. Y., Ueng, S. H., Hsueh, C., Yeh, C. T., Ho, J. Y., et al. (2018). Corylin increases the sensitivity of hepatocellular carcinoma cells to chemotherapy through long noncoding RNA RAD51-AS1-mediated

\section{DATA AVAILABILITY STATEMENT}

The original contributions presented in the study are included in the article/Supplementary Material, further inquiries can be directed to the corresponding author/s.

\section{AUTHOR CONTRIBUTIONS}

$\mathrm{OM}$ and BT conceptualized and designed the experiments. OM carried out the experiments, analyzed the data, and drafted the manuscript. KB, ES, and MW contributed to the data analysis, manuscript draft and scientific content. OM and BT revised the manuscript, contributed to the discussion and scientific content. All authors read and approved the final version of the manuscript.

\section{FUNDING}

This work was supported by the Ralph E. Hansmann Science Students Support Fund and the Sergei S. Zlinkoff Student Medical Research Fund, Hamilton College SSRF. Page charges were supported by Kirner Professorship, Hamilton College. The funders had no role in study design, data collection and analysis, decision to publish or preparation of the manuscript.

\section{ACKNOWLEDGMENTS}

We are grateful for the technical assistance and contribution of Elaine Yip.

\section{SUPPLEMENTARY MATERIAL}

The Supplementary Material for this article can be found online at: https://www.frontiersin.org/articles/10.3389/fbioe. 2020.582953/full\#supplementary-material

inhibition of DNA repair. Cell Death Dis. 9:543. doi: 10.1038/s41419-0180575-0

Chen, G., Wu, D., Guo, W., Cao, Y., Huang, D., Wang, H., et al. (2020). Clinical and immunologic features of severe and moderate coronavirus disease. J. Clin. Invest. 130, 2620-2629. doi: 10.1172/JCI137244

Chen, H., Wang, X., Yan, X., Cheng, X., He, X., and Zheng, W. (2018). LncRNA MALAT1 regulates sepsis-induced cardiac inflammation and dysfunction via interaction with miR-125b and p38 MAPK/NFкB. Int. Immunopharmacol. 55, 69-76. doi: 10.1016/j.intimp.2017.11.038

Chen, L., Xiong, J., Bao, L., and Shi, Y. (2020). Convalescent plasma as a potential therapy for COVID-19. Lancet Infect. Dis. 20, 398-400. doi: 10.1016/S1473-3099(20)30141-9

Chen, N., Zhou, M., Dong, X., Qu, J., Gong, F., Han, Y., et al. (2020). Epidemiological and clinical characteristics of 99 cases of 2019 novel coronavirus pneumonia in Wuhan, China: a descriptive study. Lancet 395, 507-513. doi: 10.1016/S0140-6736(20)30211-7

Chen, X., Zhao, B., Qu, Y., Chen, Y., Xiong, J., Feng, Y., et al. (2020). Detectable serum SARS-CoV-2 viral load (RNAaemia) is closely correlated with drastically elevated interleukin 6 (IL-6) level in critically ill COVID-19 patients. Clin. Infect. Dis. 17:ciaa449. doi: 10.1093/cid/ciaa449 
Cheng, L., Wang, P., Tian, R., Wang, S., Guo, Q., Luo, M., et al. (2019). LncRNA2Target v2.0: a comprehensive database for target genes of lncRNAs in human and mouse. Nucleic. Acids. Res 47, D140-D144. doi: 10.1093/nar/gky1051

Chu, H., Chan, J. F., Wang, Y., Yuen, T. T., Chai, Y., Hou, Y., et al. (2020). Comparative replication and immune activation profiles of SARSCoV-2 and SARS-CoV in human lungs: an ex vivo study with implications for the pathogenesis of COVID-19. Clin Infect Dis. 71, 1400-1409. doi: $10.1093 / \mathrm{cid} / \mathrm{ciaa} 410$

Costela-Ruiz, V. J., Illescas-Montes, R., Puerta-Puerta, J. M., Ruiz, C., and Melguizo-Rodriguez, L. (2020). SARS-CoV-2 infection: the role of cytokines in COVID-19 disease. Cytokine Growth Factor Rev. 54, 62-75. doi: 10.1016/j.cytogfr.2020.06.001

Cui, J., Li, F., and Shi, Z. L. (2019). Origin and evolution of pathogenic coronaviruses. Nat. Rev. Microbiol. 17, 181-192. doi: 10.1038/s41579-018-0118-9

de Wit, E., van Doremalen, N., Falzarano, D., and Munster, V. J. (2016). SARS and MERS: recent insights into emerging coronaviruses. Nat. Rev. Microbiol. 14, 523-534. doi: 10.1038/nrmicro.2016.81

Diao, B., Wang, C., Tan, Y., Chen, X., Liu, Y., Ning, L., et al. (2020). Reduction and functional exhaustion of $\mathrm{T}$ cells in patients with coronavirus disease (2019). (COVID-19). Front. Immunol. 11:827. doi: 10.3389/fimmu.2020. 00827

Djebali, S., Davis, C. A., Merkel, A., Dobin, A., Lassman, T., Mortazavi, A., et al. (2012). Landscape of transcription in human cells. Nature 489, 101-108. doi: $10.1038 /$ nature 11233

Dong, E., Du, H., and Gardner, L. (2020). An interactive web-based dashboard to track COVID-19 in real time. Lancet Infect. Dis. 20, 533-534. doi: 10.1016/S1473-3099(20)30120-1

Fatemi, R. P., Velmeshev, D., and Faghihi, M. A. (2014). De-repressing lncRNA-targeted genes to upregulate gene expression: focus on small molecule therapeutics. Mol. Ther. Nucleic. Acids 3:e196. doi: 10.1038/mtna.2 014.45

Fuchs, S. B., Lieder, I., Stelzer, G., Mazor, Y., Buzhor, E., Kaplan, S., et al. (2016). GeneAnalytics: an integrative gene set analysis tool for next generation sequencing, RNAseq and microarray data. Omics 20, 139-151. doi: 10.1089/omi.2015.0168

Guan, W. J., Ni, Z. Y., Hu, Y., Liang, W. H., He, J. X., Liu, L., et al. (2020). Clinical characteristics of coronavirus disease 2019 in China. N. Engl. J. Med. 382, 1708-1720.doi: 10.1056/NEJMoa2002032

Guaraldi, G., Meschiari, M., Cozzi-Lepri, A., Milic, J., Tonelli, R., Menozzi, M., et al. (2020). Tocilizumab in patients with severe COVID19: a retrospective cohort study. Lancet. Rheumatol. 2, e474-e484. doi: 10.1016/S2665-9913(20)30173-9

Hezroni, H., Koppstein, D., Schwartz, M. G., Avrutin, A., Bartel, D. P., and Ulitsky, I. (2015). Principles of long noncoding RNA evolution derived from direct comparison of transcriptomes in 17 species. Cell. Rep. 11, 1110-1122. doi: 10.1016/j.celrep.2015.04.023

Imam, H., Bano, A. S., Patel, P., Holla, P., and Jameel, S. (2015). The lncRNA NRON modulates HIV-1 replication in a NFAT-dependent manner and is differentially regulated by early and late viral proteins. Sci. Rep. 5:8639. doi: $10.1038 /$ srep08639

Iwakiri, J., Hamada, M., and Asai, K. (2016). Bioinformatics tools for lncRNA research. Biochim. Biophys. Acta 1859, 23-30. doi: 10.1016/j.bbagrm.2015.07.014

Jiang, Y., Liu, N., Zhu, S., Hu, X., Chang, D., and Liu, J. (2019). Elucidation of the mechanisms and molecular targets of Yiqi Shexue formula for treatment of primary immune thrombocytopenia based on network pharmacology. Front. Pharmacol. 10:1136. doi: 10.3389/fphar.2019.01136

Josset, L., Tchitchek, N., Gralinski, L. E., Ferris, M. T., Eisfeld, A. J., Green, R. R., et al. (2014). Annotation of long non-coding RNAs expressed in collaborative founder mice in response to respirartory virus infection reveals a new class of interferon-stimulated transcripts. RNA Biol. 11, 875-890. doi: 10.4161/rna.29442

Kato, Y., Sato, K., Hamada, M., Watanabe, Y., Asai, K., and Akutsu, T. (2010). RactIP: fast and accurate prediction of RNA-RNA interaction using integer programming. Bioinformatics 26, i460-i466. doi: 10.1093/bioinformatics/btq372
Kewan, T., Covut, F., Al-Jaghbeer, M. J., Rose, L., Gopalakrishna, K. V., and Akbik, B. (2020). Tocilizumab for treatment of patients with severe COVID-19: a retrospective cohort study. EClin. Med. 24:100418. doi: 10.1016/j.eclinm.2020.100418

Kiryu, H., Terai, G., Imamura, O., Yoneyama, H., Suzuki, K., and Asai, K. (2011). A detailed investigation of accessibilities around target sites of siRNAs and miRNAs. Bioinformatics 27, 1788-97. doi: 10.1093/bioinformatics/ btr276

Klijn, C., Durinck, S., Stawiski, E. W., Haverty, P. M., Jiang, Z., Liu, H., et al. (2015). A comprehensive transcriptional portrait of human cancer cell lines. Nat. Biotechnol. 33, 306-312. doi: 10.1038/nbt.3080

Kumar, S., Stecher, G., and Tamura, K. (2016). MEGA7: Molecular Evolutionary Genetics Analysis Version 7.0 for bigger datasets. Mol. Biol. Evol. 33, 18701874. doi: $10.1093 / \mathrm{molbev} / \mathrm{msw} 054$

Kurosawa, M., Numazawa, S., Tani, Y., and Yoshida, T. (2000). ERK signaling mediates the induction of inflammatory cytokines by bufalin in human monocytic cells. Am. J. Physiol. Cell. Physiol. 278, C500-C508. doi: 10.1152/ajpcell.2000.278.3.C500

Lee, S., Kopp, F., Chang, T. C., Sataluri, A., Chen, B., Sivakumar, S., et al. (2016). Noncoding RNA NORAD regulates genomic stability by sequestering PUMILIO proteins. Cell 164, 69-80. doi: 10.1016/j.cell.2015.12.017

Lei, J., Fu, Y., Zhuang, Y., Zhang, K., and Lu, D. (2019). LncRNA SNHG1 alleviates IL- $1 \beta$-induced osteoarthritis by inhibiting miR-16-5p-mediated p38 MAPK and NF-кB signaling pathways. Biosci. Rep. 39:BSR20191523. doi: 10.1042/BSR20191523

Lennox, K. A., and Behlke, M. A. (2016). Mini review on current strategies to knockdown long non-coding RNAs. J. Rare Dis. Res. Treat. 1, 66-70. doi: 10.29245/2572-9411/2016/3.1066

Letunic, I., and Bork, P. (2019). Interactive tree of life (iTOL) v4: recent updates and new developments. Nucleic Acids Res. 47, W256-W259. doi: $10.1093 /$ nar/gkz239

Li, F., Sun, J., Huang, S., Su, G., and Pi, G. (2018). LncRNA GAS5 overexpression reverses LPS-induced inflammatory injury and apoptosis through upregulating KLF2 expression in ATDC5 chondrocytes. Cell. Physiol. Biochem. 45 1241-1251. doi: 10.1159/000487455

Li, J., Ma, W., Zeng, P., Wang, J., Geng, B., Yang, J., et al. (2015). LncTar: a tool for predicting the RNA targets of long noncoding RNAs. Brief. Bioinform. 16, 806-812. doi: 10.1093/bib/bbu048

Li, Y., Li, M., Wang, M., Zhou, Y., Chang, J., Xian, Y., et al. (2020). Acute cerebrovascular disease following COVID-19: a single center, retrospective, observational study. Stroke Vasc. Neurol. 5, 279-284. doi: 10.1136/svn-2020-000431

Lippi, G., and Plebani, M. (2020). Procalcitonin in patients with severe coronavirus disease 2019 (COVID-19): a meta-analysis. Clin. Chim. Acta 505, 190-192. doi: 10.1016/j.cca.2020.03.004

Lippi, G., Plebani, M., and Henry, B. M. (2020). Thrombocytopenia is associated with severe coronavirus disease 2019 (COVID-19) infections: a meta-analysis. Clin. Chim. Acta 506, 145-148. doi: 10.1016/j.cca.2020.03.022

Liu, Y., Yang, Y., Zhang, C., Huang, F., Wang, F., Yuan, J., et al. (2020). Clinical and biochemical indexes from 2019-nCoV infected patients linked to viral loads and lung injury. Sci. China Life. Sci 63, 364-374. doi: 10.1007/s11427-020-1643-8

Lorenz, R., Bernhart, S. H., Höner zu Siederdissen, C., Tafer, H., Flamm, C., Stadler, P. F., et al. (2011). ViennaRNA package 2.0. Algorithms. Mol. Biol. 6:26. doi: 10.1186/1748-7188-6-26

Lu, Q., Ren, S., Lu, M., Zhang, Y., Zhu, D., Zhang, X., et al. (2013). Computational prediction of associations between long non-coding RNAs and proteins. BMC Genomics 14:651. doi: 10.1186/1471-2164-14-651

Ma, M., Pei, Y., Wang, X., Feng, J., Zhang, Y., and Gao, M. (2019). LncRNA XIST mediates bovine mammary epithelial cell inflammatory response via NF-кB/NLRP3 inflammasome pathway. Cell. Prolif. 52:e12525. doi: $10.1111 /$ cpr. 12525

Mallapaty, S. (2020). Animal source of the coronavirus continues to elude scientists. Nat. News doi: 10.1038/d41586-020-01449-8

Mathews, D. H., Disney, M. D., Childs, J. L., Schroeder, S. J., Zuker, M., and Turner, D. H. (2004). Incorporating chemical modification constraints into a dynamic programming algorithm for prediction of RNA secondary structure. Proc. Natl. Acad. Sci. U.S.A. 101, 7287-7292. doi: 10.1073/pnas.04017 99101 
Mehta, P., McAuley, D. F., Brown, M., Sanchez, E., Tattersall, R. S., and Manson, J. J. (2020). COVID-19: Consider cytokine storm syndromes and immunosuppression. Lancet 395, 1033-1034. doi: 10.1016/S0140-6736(20)30628-0

Morenikeji, O. B., and Thomas, B. N. (2019). In silico analyses of CD14 molecule reveal significant evolutionary diversity, potentially associated with speciation and variable immune response in mammals. Peer J. 7:e7325. doi: $10.7717 /$ peerj.7325

Okba, N. M. A., Müller, M. A., Li, W., Wang, C., GeurtsvanKessel, C. H., Corman, V. M., et al. (2020). SARS-CoV-2 specific antibody responses in COVID-19 patients. MedRxiv. doi: 10.1101/2020.03.18.20038059

Oran, D. P., and Topol, E. J. (2020). Prevalence of asymptomatic SARSCoV-2 infection: a narrative review. Ann Intern Med. 173, 362-367. doi: 10.7326/M20-3012

Ouyang, J., Zhu, X., Chen, Y., Wei, H., Chen, Q., Chi, X., et al. (2014). NRAV, a long noncoding RNA, modulates antiviral responses through suppression of interferon-stimulated gene transcription. Cell Host Microbe 16, 616-626. doi: 10.1016/j.chom.2014.10.001

Qin, C., Zhou, L., Hu, Z., Zhang, S., Yang, S., Tao, Y., et al. (2020). Dysregulation of immune response in patients with COVID-19 in Wuhan, China. Clin. Infect. Dis. 71, 762-768. doi: 10.1093/cid/ciaa248

Qin, X., Livingston, D. M., Kaelin, W. G., and Adams, P. D. (1994). Deregulated transcription factor E2F-1 expression leads to S-phase entry and p53-mediated apoptosis. Proc. Natl. Acad. Sci. U.S.A. 91, 10918-10922. doi: 10.1073/pnas.91.23.10918

Riege, K., Hölzer, M., Klassert, T. E., Barth, E., Bräuer, J., Collatz, M., et al. (2017). Massive effect on lncRNAs in human monocytes during fungal and bacterial infections and in response to vitamins A and D. Sci. Rep. 7:40598. doi: $10.1038 /$ srep40598

Roberts, T. C., Langer, R., and Wood, M. J. A. (2020). Advances in oligonucleotide drug delivery. Nat. Rev. Drug Discov. 19, 673-694. doi: 10.1038/s41573-020-0075-7

Ruan, Q., Yang, K., Wang, W., Jiang, L., and Song, J. (2020). Clinical predictors of mortality due to COVID-19 based on an analysis of data of 150 patients from Wuhan, China. Intensive Care Med. 46, 846-848. doi: 10.1007/s00134-020-05991-x

Shan, B., and Lee, W. (1994). Deregulated expression of E2F-1 induces S-phase entry and leads to apoptosis. Mol. Cell. Biol. 14, 8166-8173. doi: 10.1128/MCB.14.12.8166

Smoot, M. E., Ono, K., Ruscheinski, J., Wang, P., and Ideker, T. (2011). Cytoscape 2.8: new features for data integration and network visualization. Bioinformatics 27, 431-432. doi: 10.1093/bioinformatics/ btq675

Sun, D., Li, H., Lu, X., Xiao, H., Ren, J., Zhang, F., et al. (2020). Clinical features of severe pediatric patients with coronavirus disease 2019 in Wuhan: a single center's observational study. World J. Pediatr. 16, 251-259. doi: 10.1007/s12519-020-00354-4

Sun, H., Kamanova, J., Lara-Tejero, M., and Galán, J. E. (2018). Salmonella stimulates pro-inflammatory signalling through p21-activated kinases bypassing innate immune receptors. Nat. Microbiol. 3, 1122-1130. doi: 10.1038/s41564-018-0246-Z

Sur, S., Sasaki, R., Devhare, P., Steele, R., Ray, R., and Ray, R. B. (2018). Association between microRNA-373 and long noncoding RNA NORAD in hepatitis $\mathrm{C}$ virus-infected hepatocytes impairs weel expression for growth promotion. J. Virol. 92, e01215-e01218. doi: 10.1128/JVI.0 1215-18

Szklarczyk, D., Morris, J. H., Cook, H., Kuhn, M., Wyder, S., Simonovic, M., et al. (2017). The STRING database in 2017: quality-controlled proteinprotein association networks, made broadly accessible. Nucleic Acids Res. 45, D362-368. doi: 10.1093/nar/gkw937

Tay, M. Z., Poh, C. M., Rénia, L., MacAry, P. A., and Ng, L. F. (2020). The trinity of COVID-19: Immunity, inflammation and intervention. Nat. Rev. Immunol. 20, 363-375. doi: 10.1038/s41577-020-0311-8

Thanh Le, T., Andreadakis, Z., Kumar, A., Gómez Román, R., Tollefsen, S., Saville, M., et al. (2020). The COVID-19 vaccine development landscape. Nat. Rev. Drug Discov. 19, 305-306. doi: 10.1038/d41573-020-00073-5

Tichon, A., Gil, N., Lubelsky, Y., Solomon, T. H., Lemze, D., Itzkovitz, S., et al. (2016). A conserved abundant cytoplasmic long noncoding RNA modulates repression by pumilio proteins in human cells. Nat. Commun. 7:12209. doi: $10.1038 /$ ncomms 12209

Uhlén, M., Fagerberg, L., Hallström, B. M., Lindskog, C., Oksvold, P., Mardinoglu, A., et al. (2015). Tissue-based map of the human proteome. Science 347:1260419. doi: 10.1126/science.1260419

Uszczynska-Ratajczak, B., Lagarde, J., Frankish, A., Guigó, R., and Johnson, R. (2018). Towards a complete map of the human long non-coding RNA transcriptome. Nat. Rev. Genet. 19, 535-548. doi: 10.1038/s41576-018 -0017-y

Vaira, L. A., Salzano, G., Deiana, G., and De Riu, G. (2020). In response to anosmia and ageusia: common findings in COVID-19 patients. Laryngoscope 130:E695. doi: 10.1002/lary.28837

Vishnubalaji, R., Shaath, H., and Alajez, N. M. (2020). Protein coding and long noncoding RNA (lncRNA) transcriptional landscape in SARSCoV-2 infected bronchial epithelial cells highlight a role for interferon and inflammatory response. Genes 11:760. doi: 10.3390/genes110 70760

Wang, J., Wang, Y., Zhou, R., Zhao, J., Zhang, Y., Yi, D., et al. (2018). Host long noncoding RNA lncRNA-PAAN regulates the replication of influenza A virus. Viruses 10:330. doi: 10.3390/v10060330

Wu, C., Chen, X., Cai, Y., Zhou, X., Xu, S., Huang, H., et al. (2020). Risk factors associated with acute respiratory distress syndrome and death in patients with coronavirus disease 2019 pneumonia in Wuhan, China. JAMA Intern. Med. 180, 934-943. doi: 10.1001/jamainternmed.202 0.0994

Wu, X., and Levine, A. J. (1994). p53 and E2F-1 cooperate to mediate apoptosis. Proc. Natl. Acad. Sci. U.S.A. 91, 3602-3606. doi: 10.1073/pnas.91.9.3602

Wu, Y., Chen, C., and Chan, Y. (2020). The outbreak of COVID-19: an overview. J. Chin. Med. Assoc. 83, 217-220. doi: 10.1097/JCMA.0000000000 000270

Xiong, Y., Liu, Y., Cao, L., Wang, D., Guo, M., Jiang, A., et al. (2020). Transcriptomic characteristics of bronchoalveolar lavage fluid and peripheral blood mononuclear cells in COVID-19 patients. Emerg. Microbes Infect. 9, 761-70. doi: 10.1080/22221751.2020.1747363

Xu, X., Han, M., Li, T., Sun, W., Wang, D., Fu, B., et al. (2020). Effective treatment of severe COVID-19 patients with tocilizumab. Proc. Natl. Acad. Sci. U.S.A. 117, 10970-10975. doi: 10.1073/pnas.2005615117

Yabe, T., Sanagi, T., Schwartz, J. P., and Yamada, H. (2005). Pigment epitheliumderived factor induces pro-inflammatory genes in neonatal astrocytes through activation of NF-кB and CREB. Glia. 50, 223-234. doi: 10.1002/glia.20171

Yang, G., Lu, X., and Yuan, L. (2014). LncRNA: A link between RNA and cancer. Biochim. Biophys. Acta 1839, 1097-1109. doi: 10.1016/j.bbagrm.2014.08.012

Yang, X., Yu, Y., Xu, J., Shu, H., Liu, H., Wu, Y., et al. (2020). Clinical course and outcomes of critically ill patients with SARS-CoV-2 pneumonia in Wuhan, China: a single-centered, retrospective, observational study. Lancet Respir. Med. 8, 475-481. doi: 10.1016/S2213-2600(20)30079-5

Yang, Z., Zhao, Y., Lin, G., Zhou, X., Jiang, X., and Zhao, H. (2019). Noncoding RNA activated by DNA damage (NORAD): biologic function and mechanisms in human cancers. Clin. Chim. Acta 489, 5-9. doi: 10.1016/j.cca.2018.11.025

Ye, Q., Wang, B., and Mao, J. (2020). The pathogenesis and treatment of the cytokine storm' in COVID-19. J. Infect. 80, 607-613. doi: 10.1016/j.jinf.2020.03.037

Zhang, H., Li, H., Ge, A., Guo, E., Liu, S., and Zhang, L. (2018). Long non-coding RNA TUG1 inhibits apoptosis and inflammatory response in LPS-treated H9c2 cells by down-regulation of miR-29b. Biomed. Pharmacother. 101, 663-669. doi: 10.1016/j.biopha.2018.02.129

Zhang, L., Cheng, H., Yue, Y., Li, S., Zhang, D., and He, R. (2018). TUG1 knockdown ameliorates atherosclerosis via up-regulating the expression of miR-133a target gene FGF1. Cardiovasc. Pathol. 33, 6-15. doi: 10.1016/j.carpath.2017.11.004

Zhang, T., Wu, Q., and Zhang, Z. (2020). Probable pangolin origin of SARSCoV-2 associated with the COVID-19 outbreak. Curr. Biol. 30, 1346-1351.e2. doi: 10.1016/j.cub.2020.03.022

Zhang, X., Liu, G., Qiu, J., Zhang, N., Ding, J., and Hua, K. (2017). E2F1 regulated long non-coding RNA RAD51-AS1 promotes cell cycle progression, inhibits apoptosis and predicts poor prognosis in epithelial ovarian cancer. Sci. Rep. 7:4469. doi: 10.1038/s41598-017-04736-z 
Zhao, Y., Li, H., Fang, S., Kang, Y., Wu, W., Hao, Y., et al. (2016). NONCODE 2016: an informative and valuable data source of long noncoding RNAs. Nucleic Acids Res. 44, D203-D208. doi: 10.1093/nar/gk v1252

Zhong, Y., Yu, C., and Qin, W. (2018). LncRNA SNHG14 promotes inflammatory response induced by cerebral ischemia/reperfusion injury through regulating miR-136-5p /ROCK1. Cancer Gene. Ther. 26, 234-247. doi: $10.1038 / \mathrm{s} 41417-018-0067-5$

Zhou, P., Yang, X., Wang, X., Hu, B., Zhang, L., Zhang, W., et al. (2020). A pneumonia outbreak associated with a new coronavirus of probable bat origin. Nature 579, 270-273. doi: 10.1038/s41586-0202012-7

Ziegler, C. G. K., Allon, S. J., Nyquist, S. K., Mbano, I. M., Miao, V. N., Tzouanas, C. N., et al. (2020). SARS-CoV-2 receptor ACE2 is an interferon-stimulated gene in human airway epithelial cells and is detected in specific cell subsets across tissues. Cell 181, 1016-1035.e19. doi: 10.1016/j.cell.2020.04.035

Conflict of Interest: The authors declare that the research was conducted in the absence of any commercial or financial relationships that could be construed as a potential conflict of interest.

Copyright (c) 2021 Morenikeji, Bernard, Strutton, Wallace and Thomas. This is an open-access article distributed under the terms of the Creative Commons Attribution License (CC BY). The use, distribution or reproduction in other forums is permitted, provided the original author(s) and the copyright owner(s) are credited and that the original publication in this journal is cited, in accordance with accepted academic practice. No use, distribution or reproduction is permitted which does not comply with these terms. 